\title{
Topological characterization of neutron star crusts
}

\author{
C. O. Dorso and P. A. Giménez Molinelli \\ Departamento de Fúsica, FCEN, Universidad de Buenos Aires, Núñez, Argentina \\ J. A. López \\ Department of Physics, University of Texas at El Paso, El Paso, Texas 79968, U.S.A.
}

(Dated: October 15, 2018)

\begin{abstract}
Neutron star crusts are studied using a classical molecular dynamics model developed for heavy ion reactions. After the model is shown to produce a plethora of the so-called "pasta" shapes, a series of techniques borrowed from nuclear physics, condensed matter physics and topology are used to craft a method that can be used to characterize the shape of the pasta structures in an unequivocal way.
\end{abstract}

PACS numbers: PACS 24.10.Lx, 02.70.Ns

\section{INTRODUCTION}

Neutron stars created in the death of a massive star are composed of a dense core containing an excess of neutrons over protons, thus justifying the name. With a mass between 1 and 3 solar masses and a radius of about $10 \mathrm{~km}$, the stars are topped with a crust of about a kilometer thick where the $\beta$ decayed-produced neutrons form neutron-rich nuclear matter immersed in a sea of electrons. The crust density ranges from normal nuclear density $\left(\sim 3 \times 10^{14} \mathrm{~g} / \mathrm{cm}^{3}\right)$ at a depth of about $1 \mathrm{~km}$, to the neutron drip density $\left(\sim 4 \times 10^{11} \mathrm{~g} / \mathrm{cm}^{3}\right)$ at about $1 / 2 \mathrm{~km}$, to a light mix of neutron-rich nuclei with densities decreasing practically to zero in the neutron star envelope. Likewise the proton-to-nucleon ratio also varies from $\sim 0.25$ to $\sim 0.5$ through the crust [1], and the temperature from cold nuclear matter to about $1 \mathrm{MeV}$. The study of the structure of such crust, is the purpose of the present work.

Studies of low density nuclear matter have found that the attractive-repulsive interplay of nuclear and Coulomb forces drive low-density nuclear matter to form nonuniform structures which are collectively known as "nuclear pasta". Such arrangements go from condensed phases with voids filled with nuclear gas, to "lasagnalike" layers of nuclei surrounded by gas, to "spaghettilike" rods embedded in a nuclear gas, to ever decreasing "meatball-like" clumps which practically dissolve into a gaseous phase [2].

Early investigations have used static models which rely mostly on energy considerations to determine the structures that are most energetically favorable. Among the various theories proposed, the ones used most recurrently are the Compressible Liquid Drop Model [3 [5], the Extended Thomas-Fermi Model 2, 6 8], and the HartreeFock method [5, 9-13].

On the other hand, there are dynamical models that go beyond mean fields to predict the formation of the pasta phases as an asymptotic equilibrium state resulting from an evolution of a dynamical system. The most used methods are the semiclassical molecular dynamics 14
16] and the quantum molecular dynamics [17 22].

On the general composition of the pasta, most models agree on the formation of varying structures at subnormal densities but not on how the sequence the phases arises. Since the physical mechanism responsible for the phase transition pattern is a subtle interplay between Coulomb and nuclear energies which varies only a few $\mathrm{keV} / \mathrm{fm}^{3}$ between phases, the precise transition pattern is easily altered by the ingredients of the theoretical models. The fact that the Coulomb interaction between the electron sea and the nucleons -whose screening effect stabilizes the overall system modifying its structure- must be treated under different approximations in different models 22] complicates any cross-model comparison even more.

An additional problem which makes comparisons between models difficult or impossible in some cases, is the lack of a quantifiable characterization of different pasta phases. The identification of phases has been done mainly through visual inspections of snapshots of spatial nucleon distributions obtained from calculations [23]. The QMD approach of reference [17], for example, produces nuclear holes, slabs, cylinders and spheres similar to those predicted by the Thomas Fermi model [2, 6, 7], but at different densities and temperatures.

In spite of this, the pasta phases have been characterized globally. For instance, static models have been used to calculate average densities [8] and volume fractions of the different phases 24]. Pasta bulk properties, such as the shear viscosity [25] and diffusion coefficients [26] have also been obtained using molecular dynamics simulations. Refined studies have used radial correlation functions to characterize the nucleon distributions [19] and the pasta structure factor to study charge density fluctuations [27].

More recently, shape characterizations were attempted both in dynamical simulations and with static models; the former use topological measures such as the Minkowski functionals and the Euler characteristics [23], while the latter modified the liquid drop model with a curvature correction to detect structure shape changes [28].

Thus the motivation of the present study: how to 
achieve a precise enough characterization of the pasta phases? What property can be used to signal a change of pasta phase? The purpose of the present work is to construct the instruments needed to properly quantify the pasta structures.

Taking advantage of the microscopic details produced by a classical molecular dynamics model, this investigation combines the power of cluster detection algorithms used in nuclear collisions with indicators borrowed from condensed matter physics and topology to detect the transitions between pasta phase structures in a quantitative way.

After an introduction of the model, we will introduce a series of techniques used to classify the pasta structures that will help us reach our goal. Starting from global measures to understand the cluster composition (fragment size distribution, nucleon mobility and persistence, fragment isotopic composition and radial distribution function), we will progress into topological tools (Minkowski functionals) that will allow us to characterize the shape of the pasta structures as well as to detect changes between them. A final discussion of the results will then help us reach a series of conclusions and to draw an outlook of the future tasks.

\section{NUCLEON DYNAMICS}

To study the structure of stellar crusts is necessary understand the behavior of nucleons at the proper densities, temperatures and proton-to-neutron ratios; such knowledge comes from the study of heavy ion fragmentations. The initial statistical studies of nuclear collisions of the 1980's [29, 30], rapidly gave way to dynamical theories based on classical, semiclassical and quantum approaches.

The semiclassical models use the Boltzmann-UehlingUhlenbeck equations [31] to track the time evolution in phase space of the probability of finding a particle moving in a mean field. On the other hand, the quantum molecular dynamics models $(Q M D)$ solve the equations of motion of nucleon wavepackets moving within mean fields. Unfortunately, these theories either do not lead to cluster formation or yield a poor description of cluster properties and both must resort to the use of all sorts of extraneous techniques such as adding fragments by hand, coupling to "afterburners" to produce secondary decays [32], and introducing hidden adjustable parameters (e.g. width of wavepackets, number of test particles, modifications of mean fields, effective masses and cross sections, etc.) to satisfy the operator's taste.

These problems are either non-existent or much reduced in classical models. Classical dynamical models generally solve Newton's equations of motion to track individual nucleons moving under two-body potentials; coupled to cluster recognition algorithms these calculations yield microscopic views of nuclear reactions as well as of nuclear structures. The only apparent disadvantage of the classical models would be the lack of quantum effects, such as the Pauli blocking; fortunately, in stellar environments the very small nucleon energies lead to frozen-like structures where the blocking of momentumtransferring collisions ceases to be relevant. [See Ref. 33] for a calculation of the mean thermal wavelength of a nucleus in stellar conditions to justify the use of a classical approach.]

Let this rather long preamble serve to justify extending the use of a classical model designed for nuclear reactions to the study neutron star crusts. In this work we use a classical model to obtain a detailed microscopic picture of the pasta structures and be able to detect transitions between phases.

\section{A. Classical Molecular Dynamics}

We use a molecular dynamics code combined with algorithms for cluster recognition. Our classical molecular dynamics model, $C M D$ [34], is based on the pioneering work of Pandharipande [35] and has been very fruitful in nuclear studies of, among other phenomena, neck fragmentation 36], phase transitions 37, 38], critical phenomena [39, 40], the caloric curve [41, 42], and isoscaling [43, 44] all without any adjustable parameters. Readers are directed to these references for further details on the model; here only a brief synopsis will be presented along with its extension to infinite systems.

In a nutshell, $C M D$ treats nucleons as classical particles interacting through a two-body potential and solves the coupled equations of motion of the many-body system to obtain the time evolution of all particles. Since the $(\mathbf{r}, \mathbf{p})$ information is known for all particles at all times, it is possible to know the structure of the nuclear medium from a microscopic point of view.

$C M D$ uses the phenomenological potentials developed by Pandharipande [35]:

$$
\begin{aligned}
V_{n p}(r)= & V_{r}\left[\exp \left(-\mu_{r} r\right) / r-\exp \left(-\mu_{r} r_{c}\right) / r_{c}\right] \\
& -V_{a}\left[\exp \left(-\mu_{a} r\right) / r-\exp \left(-\mu_{a} r_{a}\right) / r_{a}\right] \\
V_{N N}(r)= & V_{0}\left[\exp \left(-\mu_{0} r\right) / r-\exp \left(-\mu_{0} r_{c}\right) / r_{c}\right],
\end{aligned}
$$

where $V_{n p}$ is the potential between a neutron and a proton and it is attractive at large distances and repulsive at small ones, and $V_{N N}$ is the interaction between identical nucleons and it is purely repulsive. Notice that no bound state of identical nucleons can exist, also notice that, at a difference from potentials used by other models [15], these potentials have a hard core.

The cutoff radius is $r_{c}=5.4 \mathrm{fm}$ after which the potentials are set to zero. Two sets of values for the Yukawa parameters $\mu_{r}, \mu_{a}$ and $\mu_{0}$ were fixed by Pandariphande to correspond to infinite-nuclear matter systems with an equilibrium density of $\rho_{0}=0.16 \mathrm{fm}^{-3}$, a binding energy $E\left(\rho_{0}\right)=16 \mathrm{MeV} /$ nucleon and compressibility of about $250 \mathrm{MeV}$ ("Medium") or $535 \mathrm{MeV}$ ("Stiff") 35]. In the past, a combination of Monte Carlo and molecular 


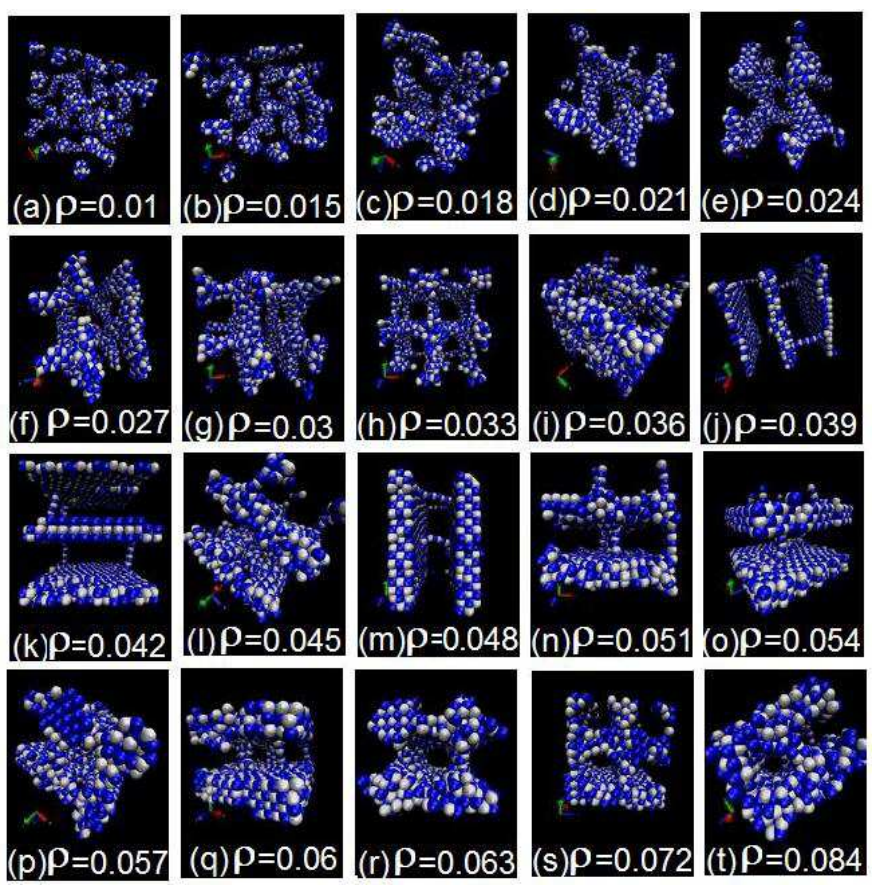

FIG. 1: (Color online) Smörgåsboard of pasta shapes corresponding to the densities shown and to $x=0.5$ and $T=0.1 \mathrm{MeV}$.

dynamics techniques was applied within a statistical formalism to obtain neutron start crust properties [15].

\section{B. Simulating the neutron star crust}

To study the neutron start crust we use $C M D$ to simulate an infinite medium. Systems with 2000 or 3000 nucleons were constructed and replicated with periodic boundary conditions in 26 surrounding cells. In particular, the proton ratios used were of $x=Z / A=0.5$ (1000 neutrons and 1000 protons) or 0.3 (2000 neutrons and 1000 protons). The cubical box size used was adjusted as to achieve densities between $\rho=0.01 \mathrm{fm}^{-3}$ (about $\left.\rho_{0} / 15\right)$ and $\rho_{0}$.

As the crust is expected to be embedded in a degenerate electron gas produced by weak decays during the supernova explosion, it is necessary to take into account its Coulomb interaction. Although the nucleon-electron system is overall neutral and $\beta$-equilibrated, the infinite Coulomb range requires the use of some approximation; two common approaches are the Thomas-Fermi screened Coulomb potential (used in $Q M D$ in 17]) or the Ewald summation procedure [45]. Although $C M D$ is able to operate under either approximation, in this work the former is adopted (see [46] for a comparison of methods under $C M D)$.

Approximating the electron gas as a uniform ideal Fermi gas at the same number density as the protons, its effect can be included in the nucleon's equations of

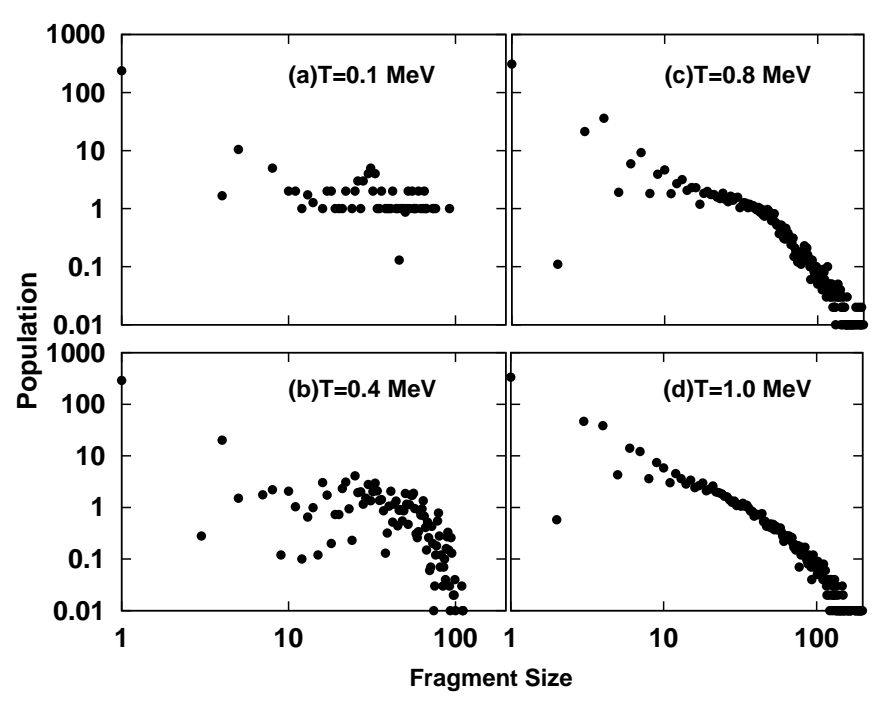

FIG. 2: Temperature evolution of the fragment size distribution obtained from 200 configurations with $x=0.3$ and $\rho \approx \rho_{0} / 10$.

motion by means of the screened Coulomb potential obtained from the Poisson equation:

$$
V_{C}^{(S c r)}(r)=\frac{e^{2}}{r} \exp (-r / \lambda)
$$

where the relativistic Thomas-Fermi screening length is: $\lambda=\left(\pi^{2} / 2 e\right)\left[k_{F}^{2}\left(k_{F}^{2}+m_{e}^{2}\right)\right]^{-\frac{1}{4}}, m_{e}$ is the electron mass, $k_{F}=\left(3 \pi^{2} \rho_{e}\right)^{1 / 3}$ is the electron Fermi momentum, and $\rho_{e}$ is the electron gas number density equal to that of the protons. The size of the simulation cell, $L=(A / \rho)^{1 / 3}$, should be significantly larger than $\lambda$; in our case we satisfy such requirement using the prescription of [15] and setting $\lambda=10 \mathrm{fm}$.

The trajectories of individual nucleons, now governed by the Pandharipande and the screened Coulomb potentials, are then tracked using a Verlet algorithm with energy conservation of $\mathcal{O}(0.01 \%)$. The system is forceheated or cooled using isothermal molecular dynamics with the Andersen thermostat procedure [47] which gradually cools in small temperature steps while reaching thermal equilibrium at every step. We focus in the range of $T=0.1$ to $1.0 \mathrm{MeV}$; although this last temperature is large for stellar crusts, in terms of the nucleon dynamics it practically corresponds to a frozen state.

\section{CHARACTERIZING THE CRUST}

At a difference from most $Q M D$ simulations, which tend to track individual evolutions, here we obtain reliable statistics by sampling 200 times each configuration 


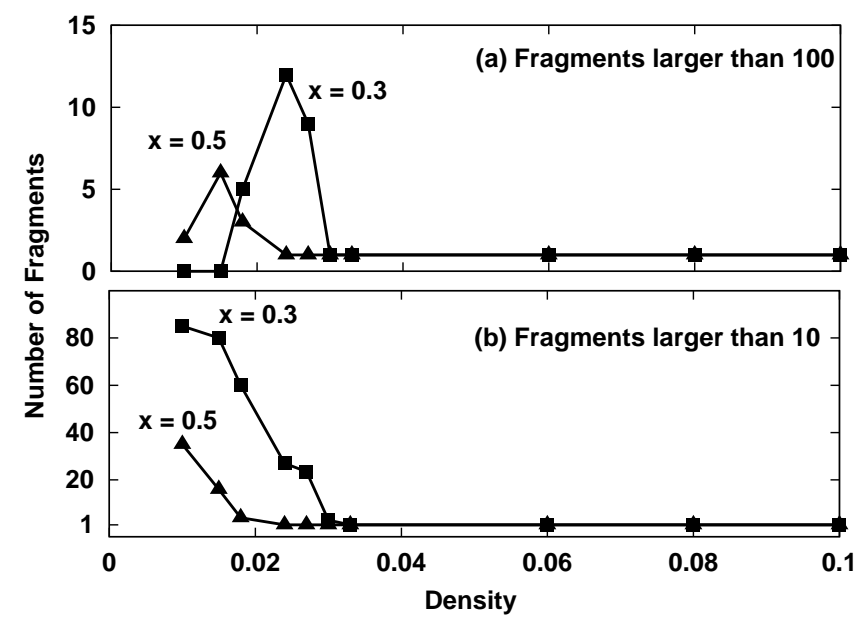

FIG. 3: Relative abundance of large clusters as a function of density for $x=0.3$ and 0.5 at $T=0.1 \mathrm{MeV}$.

with specific $x, \rho$, and $T$ conditions. Figure 1 shows an smörgåsboard of Italian delicacies produced by $C M D$ with $x=0.5, T=0.1 \mathrm{MeV}$ and twenty different densities; please notice that for clarity the figures do not show single nucleons, i.e. the gaseous phase. In spite of their beauty, one cannot use those figures to properly characterize the pasta shapes, for that one must resort to other, less visually attractive, techniques.

On each of the configurations achieved, the nucleon $(\mathbf{r}, \mathbf{p})$ information is recorded and used later to identify clusters and to characterize the structure by means of the liquid structure function and the Minkowski functionals.

\section{A. Cluster composition}

The nucleon positions are used to identify clusters by means of the "Minimum Spanning Tree" (MST) algorithm refined for nucleon dynamics in [48, 49]. In summary, $M S T$ looks for correlations in configuration space: a particle $i$ belongs to a cluster $C$ if there is another particle $j$ that belongs to $C$ and $\left|\mathbf{r}_{i}-\mathbf{r}_{j}\right| \leq r_{c l}$, where $r_{c l}$ is a clusterization radius which is set to $3.0 \mathrm{fm}$. In spite of using only $r$-space correlations, $M S T$ yields accurate results in the case of stellar crusts due to the low temperatures and small momentum transfer, and thus here it is preferred over other more robust cluster-detection algorithms (such as the "Early Cluster Recognition Algorithm", ECRA [50], which take into account relative momenta and binding energies). In our case of periodic boundary conditions, the MST method was modified to recognize fragments that extend into adjacent cells.

Figure 2 shows examples of the fragment population obtained with $C M D-M S T$ at $x=0.3, \rho=0.015 \mathrm{fm}^{-3}$

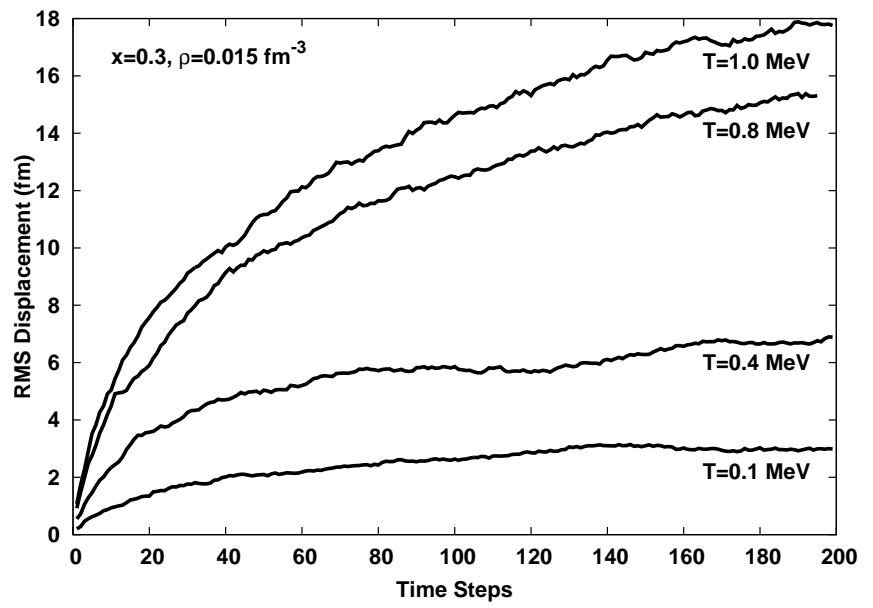

FIG. 4: Root mean square displacement of the nucleons from their original position as a function of the simulation time steps for systems with $\rho=0.015 \mathrm{fm}^{-3}$ and $x=0.3$ at the listed temperatures.

and four different temperatures; of particular interest is the evolution of the clustering as a function of the temperature as it shows a trend opposite to that observed in nuclear collisions. The figure shows a typical evolution for the range $0.1 \mathrm{MeV}<T<0.1 \mathrm{MeV}$ and, as it can be clearly observed, the large fragment multiplicity increases with $T$. This is in opposition to what happens in heavy ion reactions at high energies where heavier fragments shrink in size by particle evaporation during the final expansion stage of the reaction. In the case of infinite systems, however, the lack of expansion (and lack of a reduced pressure) makes evaporation less probable and, combined with the possibility of connecting fragments to neighboring cells, it favors the growth of cluster sizes as soon as the nucleons reach enough mobility with increasing $T$.

The growth of large fragment multiplicity can also be seen as a function of the density. Figure 3 shows a typical behavior of the relative multiplicity of large clusters, $A>100$ and $A>10$, obtained at different densities for both $x=0.3$ and 0.5 at $T=0.1 \mathrm{MeV}$. As the density increases, the number of clusters of $A>100$ increases practically linearly with $\rho$ up until a single large fragment is formed; smaller clusters of $A>10$ are abundant at low densities but decrease for larger densities. The density at which the number of large clusters condense into a single one can be thought of as a "percolation" density, this value, of course, depends on the simulation parameters such as number of particles, cell size, temperature, etc.; for the cases shown, the percolation densities are $\rho \approx 0.03 \mathrm{fm}^{-3}$ for $x=0.3$ and $\rho \approx 0.024 \mathrm{fm}^{-3}$ for $x=0.5$ at $T=0.1 \mathrm{MeV}$.

The dynamics of the nucleons within systems in equilibrium can be gauged through their average displacement as a function of "time", i.e. through the time steps 


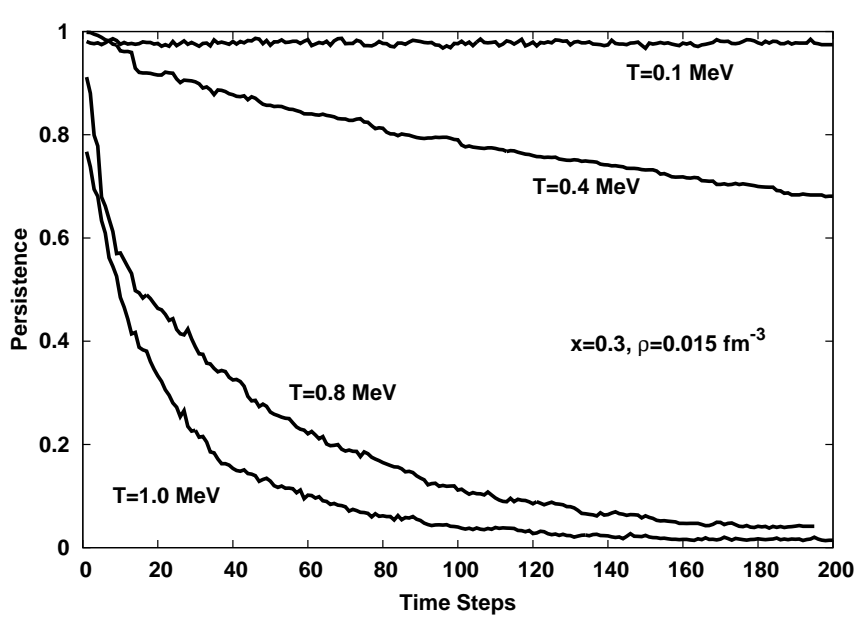

FIG. 5: Time evolution of the persistency for the listed temperatures as a function of the simulation time steps for $x=0.3$ and $\rho=0.015 \mathrm{fm}^{-3}$.

of the simulation. Figure 4 shows the root mean square displacement of the nucleons from their original position in 200 time steps; as a metric one must remember that the range of the potential is $5.4 \mathrm{fm}$ and -as we will see in section III B - the interparticle distance at these densities is of the order of $1.7 \mathrm{fm}$. The increment in mobility as a function of the temperature is obvious.

Likewise, the microscopic stability of the clusters can be quantified through the "persistency" [51, 52] which measures the tendency of members of a given cluster to remain in the same cluster. Figure 5 shows the time evolution of the persistency for systems with $\rho=0.015 \mathrm{fm}^{-3}$ and $x=0.3$ at the listed temperatures; notice that a persistency of $\sim 1$ indicates that most of the particles remain in the same cluster, while smaller values indicate a larger exchange rate. The anti-correlation between this and the previous figure is clear, more mobility implies less persistency, and viceversa.

Another interesting descriptor is the isospin content of the clusters produced. By keeping track of the number of protons and neutrons on each fragment it is possible to determine the $x$ value for each cluster. An example of this is shown in figure 6 where the $x$ content of the fragments is plotted as a function of the mass of the clusters obtained at a density of $\rho=0.015 \mathrm{fm}^{-3}$ and with $x=0.5$ (top two plots) and $x=0.3$ (bottom four). Several effects are noticeable: small clusters $(A \lesssim 10)$ tend to have less protons than the average resulting in smaller $x$ values; for the case of $x=0.3$ there is a prominent binding of one proton to two neutrons which results in clusters of all sizes with values of $x \approx 1 / 3$; the previous effect is not present in the case of $x=0.5$ in which all the cluster maintain their $x$ values around $1 / 2$.

We close this subsection noticing that, in spite of being a good indicator of the percolating density, the cluster multiplicity is a poor descriptor of the pasta shapes.

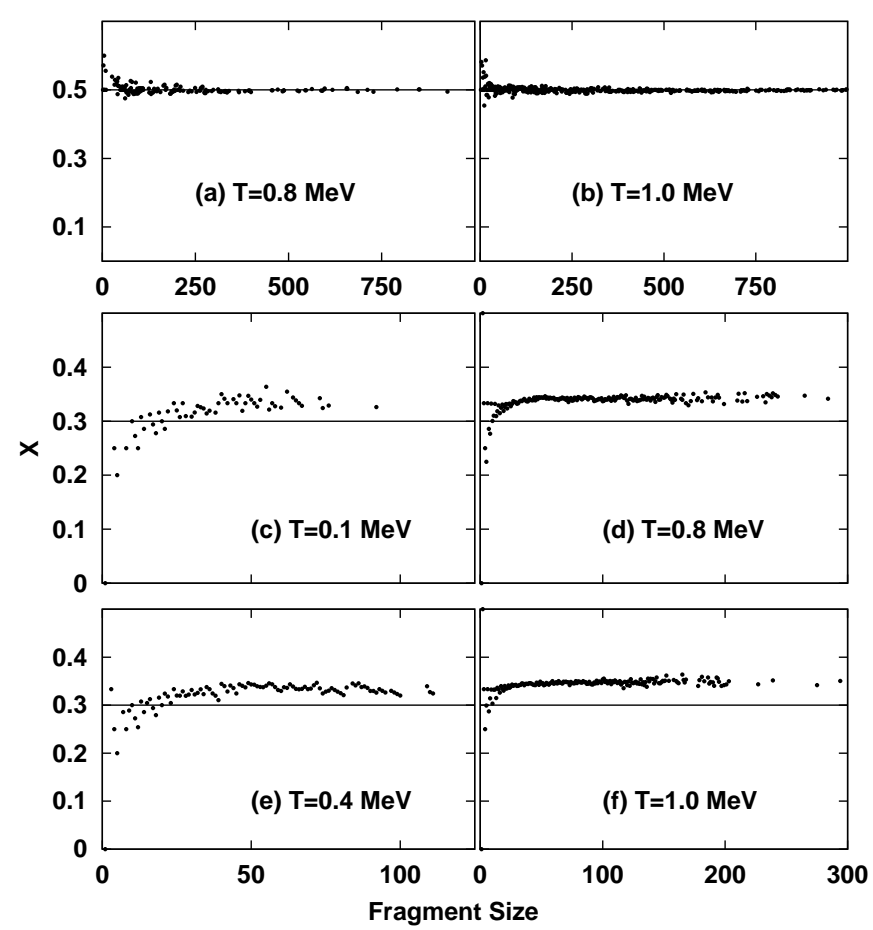

FIG. 6: Example of the $x$ value $(x=p / A)$ of the clusters versus their size, corresponding to systems with $\rho=0.015 \mathrm{fm}^{-3}$ and $x=0.5$ (top two plots) and $x=0.3$ (bottom four plots) at the temperatures listed.

Stepping up in complexity, we now turn to the radial correlation function to probe the pasta al dente.

\section{B. Pair correlation function}

Another global characterization of the structure of nuclear matter is obtained from the pair correlation function, $g(r)$, which is the ratio of the average local density to the global density, $g(r)=\rho(r) / \rho_{0}$; it gives information about the spatial ordering of the nuclear medium.

For computing purposes, the pair correlation function $g(r)$ is taken as the conditional probability density of finding a particle at $\mathbf{r}_{i}+\mathbf{r}$ given that there is one particle at $\mathbf{r}_{i}$. Formally,

$$
g(r)=\frac{V}{4 \pi r^{2} N^{2}}\left\langle\sum_{i \neq j} \delta\left(r-r_{i j}\right)\right\rangle,
$$

where $r_{i j}$ is $\left|\mathbf{r}_{i}-\mathbf{r}_{j}\right|$. For our case, this was calculated by constructing histograms of the distances between particles for several configurations obtained with the same $x$, $\rho$ and $T$ and then averaging them; to include all particles and their images the range was extended to $r_{i j} \leq 1.5 \mathrm{~L}$. 


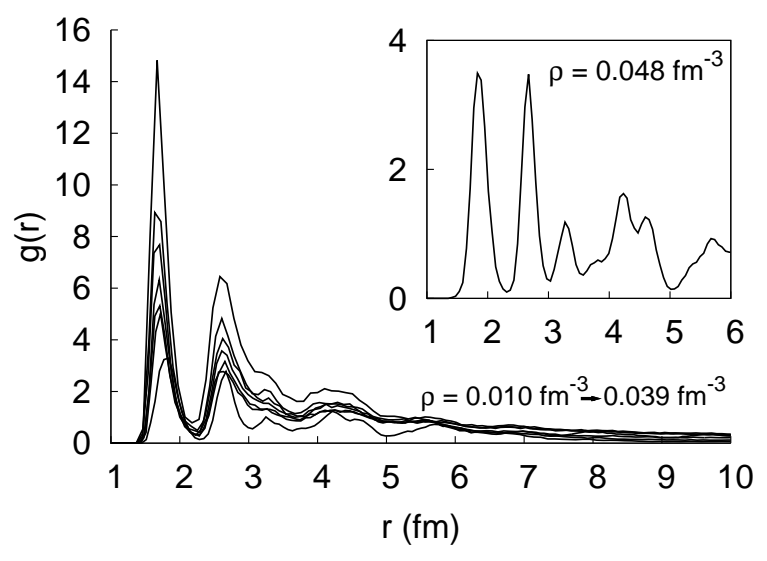

FIG. 7: Examples of the radial correlation function for $x=$ $0.5, T=0.1 \mathrm{MeV}$ and various densities. Also shown is the case when nearest neighbors are as probable as second nearest neighbors indicating a "lasagna" type structure; $r$ is in $f m$.

Figure 7 shows examples of $g(r)$ obtained for cases with 2, 000 nucleons, $x=0.5, T=0.1 \mathrm{MeV}$ and densities $0.01<\rho<0.039 \mathrm{fm}^{-3}$. The inset shows the case $\rho=0.048 \mathrm{fm}^{-3}$ when the nearest neighbors are just as probable as second nearest neighbors signalling the onset of a "lasagna" type structure, cf. Fig. 1.

It is worth noticing that, in the case shown, the location of the nearest neighbors remains constant at $r \sim$ $1.7 \mathrm{fm}$ at all densities. This is due to the fact that -since at subcritical densities the medium is metastable or unstable, it breaks into a gaseous and a condensed phasethe position of the peak of $g(r)$ is an average between the location of neighbors in the gas-liquid mixture; the condensed matter at normal density has nearest neighbors at $r \approx 1.4 \mathrm{fm}$ 35.

Once again, in spite of the rich information derived from $g(r)$, it is still insufficient to tag the phases unequivocally; for this, other more complex constructs must be borrowed from cosmology and, ultimately, from topology.

\section{Topological constructs}

The most obvious properties of closed surfaces that can be used to characterize their shapes are the volume $V$, surface area $A$, and the curvature. The latter is less trivial than the other two as it does not hold a unique value for a given shape but varies from point to point; however, mean curvatures of a closed body can be obtained by an averaging procedures such as the "integral mean curvature", defined as $H=\int d f\left(R_{1}+R_{2}\right) / 2 R_{1} R_{2}$ where $R_{1}$ and $R_{2}$ are the principal radii of curvature of the surface and $d f$ is a differential of area.

In general, $V, A, H$ plus an interesting construct known as the Euler characteristic are collectively known as the "Minkowsky functionals"; according to integral ge-
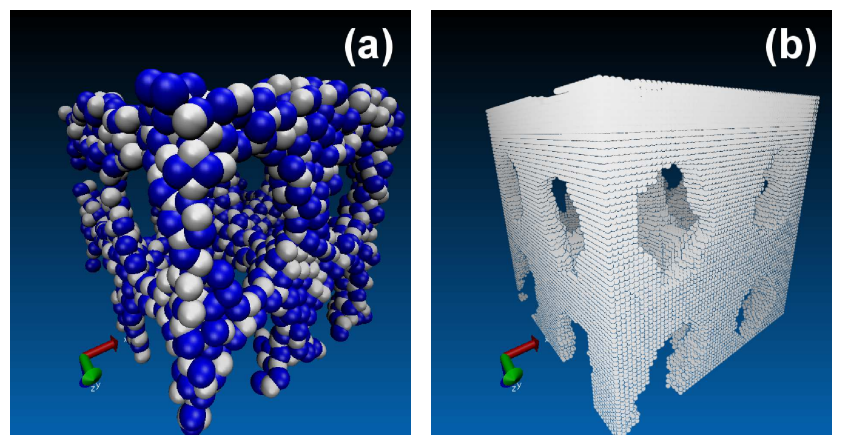

FIG. 8: (Color online) Sample transformation of a nuclear structure to a corresponding polyhedron. The structure corresponds to a case with $x=0.5, \rho=0.33 \mathrm{fm}^{-3}$ and $T=0.1 \mathrm{MeV}$.
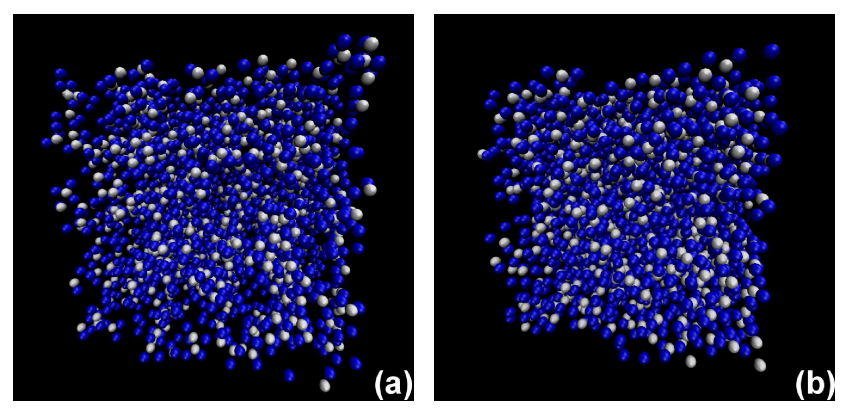

FIG. 9: (Color online) Spatial configurations formed under $T=0.4$ and $\rho=0.045 \mathrm{fm}^{-3}$ (left) and $T=1.0$ and $\rho=$ $0.072 \mathrm{fm}^{-3}$ (right), both for $x=0.3$.

ometry the morphological properties of $3 D$ objects can be completely described in terms of them.

The XVIII century work of Euler-L'Huilier showed that, independent of the shape of any polyhedra, when deducting the number of edges from the number of vertices and adding the number of faces it always yields 2 plus twice the number of cavities, quantity now known as the "Euler characteristic", $\chi$. Although this previous property is for solids bounded by plane surfaces, it also applies in any $3 D$ surfaces with $\chi$ related to the total curvature of the surface through the Gauss-Bonnet theorem.

In topological terms, two orientable closed surfaces are homeomorphic to each other if their Euler characteristics are the same. Conversely, two homomeorphic closed surfaces will always have the same value of $\chi$. Therefore, since our pasta niblets are all orientable, their Euler characteristic completely classifies them up to an homeomorphism; adding the rest of the Minkowsky functionals eliminates such redundancy and guarantees a complete classification of the pasta shapes.

An immediate problem is the fact that the nuclear clusters are not polyhedra and do not even form closed surfaces. This obstacle, however, can be circumvented by 

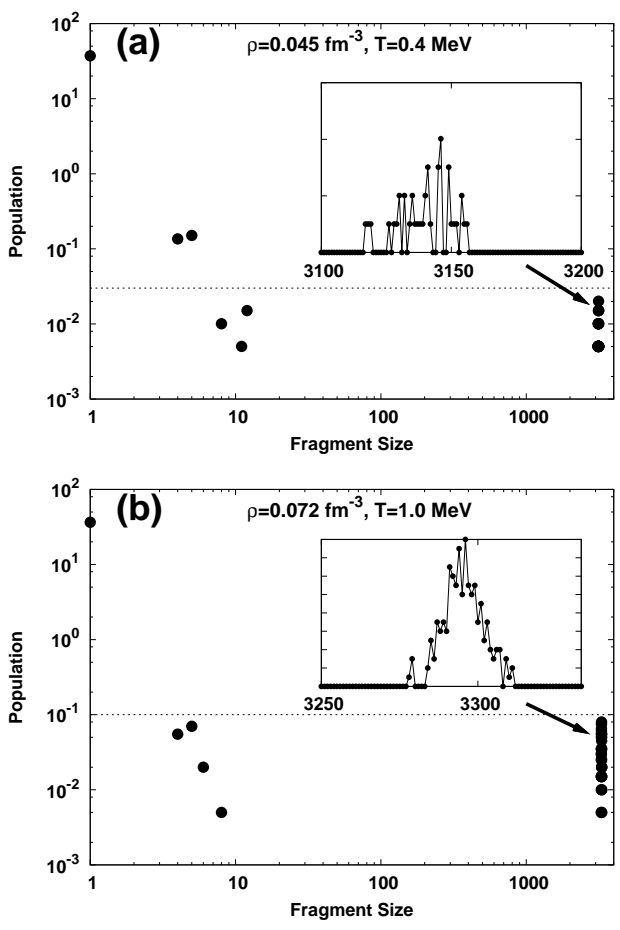

FIG. 10: Multiplicites corresponding to figure 9 although not identical they are very similar.

replacing the nuclear structure with a scaffold-like armature composed of cells enclosing each one nucleon. In our case this is done through the algorithm of Michielsen and De Raedt [53] which has already been used in the study of stellar crusts albeit in a different methodology 21].

Synoptically, the simulation volume is subdivided into a mall of cubic cells. Those cells which contain the coordinates of a nucleon are kept while the rest are deleted. The sizes of the cells are made smaller than the nearest neighbor distance found in $g(r)$ to enforce a one-particleper-cell occupation, but not too small as to avoid creating spurious cavities between neighboring nucleons. It is on this imaginary platform that the Minkowsky functionals of the nuclear structure are computed.

In general, $\chi$ equals the number of regions of connected grid cells minus the number of completely enclosed regions of empty grid cells. Two grid cells are connected if they are immediate neighbors, next-nearest neighbors, or are connected by a chain of occupied grid cells. Characterizing the connected structure by its number of occupied cubes, $n_{c}$, edges, $n_{e}$, faces, $n_{f}$, and vertices, $n_{v}$, including possible contributions from the interior of the structure, the Minkowski functionals can be calculated through 53

$$
\begin{aligned}
V=n_{c}, & A=-6 n_{c}+2 n_{f}, \\
2 B=3 n_{c}-2 n_{f}+n_{c}, & \chi=-n_{c}+n_{f}-n_{e}+n_{v}
\end{aligned}
$$

where $V$ stands for the volume, $A$ for the area, $B$ for the mean breadth $B$ and $\chi$ for the Euler number; the mean

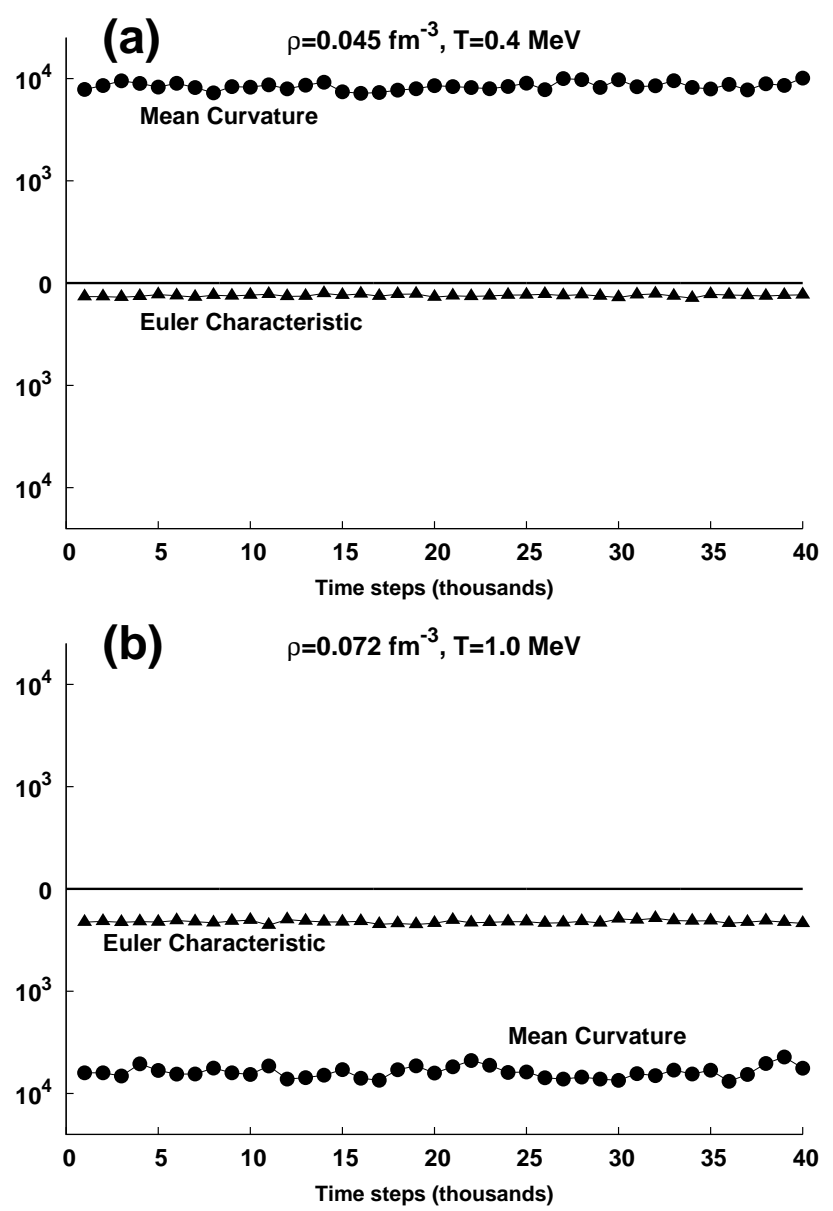

FIG. 11: Euler and curvature of the structures shown in figure 9. the difference between the two cases is easy to spot.

breadth measures of the average "size" of a body and it is related to the integral mean curvature $H$ mentioned before. Figure 8 shows a typical nuclear structure along with the grid constructed around it; the values of the Minkowski functionals obtained from such grid are Curvature $=215$ and Euler $=-17$, as we will see next such shape can be classified as a "jungle gym".

\section{Topological classification of the pasta}

To illustrate the use of topology to classify the pasta shapes let us use two seemingly similar structures obtained with $x=0.3$ but at different densities and temperatures, namely $\rho=0.045 \mathrm{fm}^{-3}$ and $T=0.4 \mathrm{MeV}$ and $\rho=0.072 \mathrm{fm}^{-3} \mathrm{~T}=1.0 \mathrm{MeV}$. The spatial configurations of these two cases, practically identical to the eye, are presented in figure 9. Although there are minor differences (see insets), figure 10] shows that both configurations have very similar mass multiplicities.

The difference between the configurations, however, surfaces when we calculate the corresponding curvature 

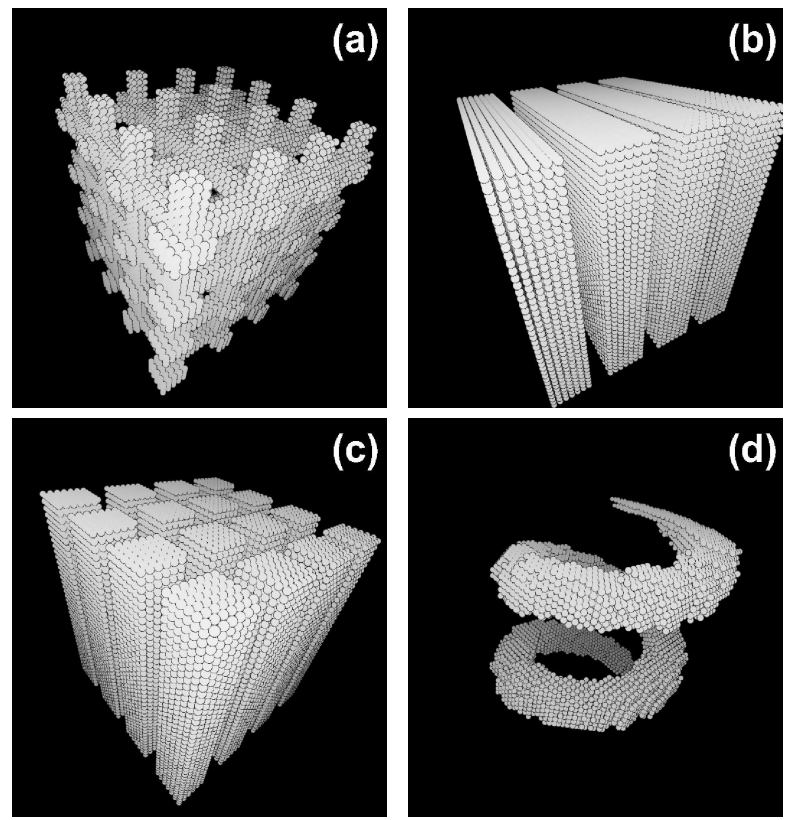

FIG. 12: (Color online) Typical artificial "pastas" used to test the classification powers of the Euler-Curvature combination. Respectively they are the "jungle gym" (top left), "lasagnas" (top right), "straight spaghettis" (bottom left) and a "curled spaghetti".

and Euler numbers. Figure 11] shows the evolution of the curvature and of the Euler number as the simulations advances after equilibration. Clearly shown are the inverted values of Euler and the curvature for the two cases; while the curvature is positive (with less cavities than bellies) and large in the low $T$ low $\rho$ case, it becomes negative (with more internal cavities than tummies) and smaller in the opposite case.

To investigate this point further, we created artificial structures in the form of gnocchi, spaghetti, lasagna and crossed-lasagnas, which we call "jungle gym", and their inverse structures (with voids replacing particles and viceversa) and calculated the values of the two topological variables; some of the structures used are shown in figure 12 and their location in the Curvature-Euler plane in figure 13. The magnitudes shown are determined by the size of the structure as well as the digitization cell size. In general one can conclude that lasagnas tend to lie near the origin, spaghettis have near zero Euler numbers and positive curvatures, gnocchis have positive curvatures and Euler numbers, and "jungle gyms" positive Euler number and negative curvature; all anti-structures reverse the curvature but maintain the Euler characteristic. All cases calculated at all $x$ values, densities and temperatures were observed to satisfy this classification.

For instance, the structure in figure 8 with curvature 215 and Euler number -17 is clearly a "jungle gym"; it must be remarked that -to our knowledge- this is the first time this type of structure has been reported. Like-
TABLE I: Classification Curvature - Euler

\begin{tabular}{|c|c|c|c|c|c|c|}
\hline $\begin{array}{l}\text { Density } \\
\left(f m^{-3}\right)\end{array}$ & $\begin{array}{r}x \\
\text { Curvature }\end{array}$ & $\begin{array}{l}=0.5 \\
\text { Euler }\end{array}$ & Topology & ${ }^{x}$ & $\begin{array}{l}=0.3 \\
\text { Euler }\end{array}$ & Topology \\
\hline 0.01 & (a) 100 & 100 & G & (A) 96 & 27 & $\mathrm{G}$ \\
\hline 0.015 & 73 & 50 & G & 92 & 7 & G-S \\
\hline 0.018 & 58 & 17 & G-S & 79 & -9 & $\mathrm{~S}$ \\
\hline 0.021 & 36 & -25 & S-J & & & \\
\hline 0.024 & 22 & -28 & S-J & 58 & -18 & J-S \\
\hline 0.026 & & & & 51 & -39 & $\mathrm{~J}$ \\
\hline 0.027 & 9 & -42 & $\mathrm{~J}-\mathrm{L}$ & 47 & -37 & $\mathrm{~J}$ \\
\hline 0.03 & 10 & -39 & $\mathrm{~J}-\mathrm{L}$ & 48 & -7 & S \\
\hline 0.033 & 9 & -47 & $\mathrm{~J}$ & 18 & -75 & $\mathrm{~J}$ \\
\hline 0.036 & 8 & -42 & $\mathrm{~J}$ & & & \\
\hline 0.039 & -11 & -6 & L-AJ & & & \\
\hline 0.042 & -15 & -8 & L-AJ & & & \\
\hline 0.045 & 1 & -33 & L-J & -54 & -100 & AJ \\
\hline 0.048 & -5 & -11 & L & & & \\
\hline 0.051 & -7 & -17 & AS-AJ & -94 & -41 & AJ \\
\hline 0.054 & -1 & -11 & L-AJ & & & \\
\hline 0.057 & -9 & -30 & AJ & & & \\
\hline 0.06 & -9 & -17 & AJ & -100 & 66 & $\mathrm{AG}$ \\
\hline 0.063 & -10 & -30 & AJ & & & \\
\hline 0.072 & -12 & -8 & AS-AJ & (L) -60 & 90 & $\mathrm{AG}$ \\
\hline 0.084 & (t) -19 & -8 & AJ & & & \\
\hline
\end{tabular}

wise, the structure in the left panel of figure 9, with both negative curvature and Euler number, can be classified as an "anti-jungle gym", whereas the accompanying structure with positive curvature and negative Euler number would have to be classified as a "jungle gym".

Table \shows the classification of several of the structures obtained in our study at $T=0.1 \mathrm{MeV}$ and at the listed densities, and figure 14 shows their location in the $C-E$ plane; the $x=0.5$ column of the table and the circular dots on the figure correspond to the structures presented in figure 1 As the absolute magnitude of the curvature and Euler number depends on the overall size of the structure, i.e. on the number of particles used, the data in table \ were normalized to have maximum absolute values of 100 . In the table, the classifications are abbreviated as $\mathrm{G}$ for gnocchi, $\mathrm{J}$ for jungle gym, L for lasagna, S for spaghetti, and AG, AJ, AL and AS for the reverse structures.

\section{CONCLUDING REMARKS}

And thus we have reached our objective. The combination of curvature and Euler number and a proper recognition of fragments appears to be robust enough as to uniquely classify the shapes attained by the nucleons at densities, temperatures and isospin content of interest in the study of neutron star crusts. In obtaining this result, the classical molecular dynamics and associated tools (cluster recognition algorithms, persistence, etc.) proved to be a valuable method, which we now plan to exploit. 


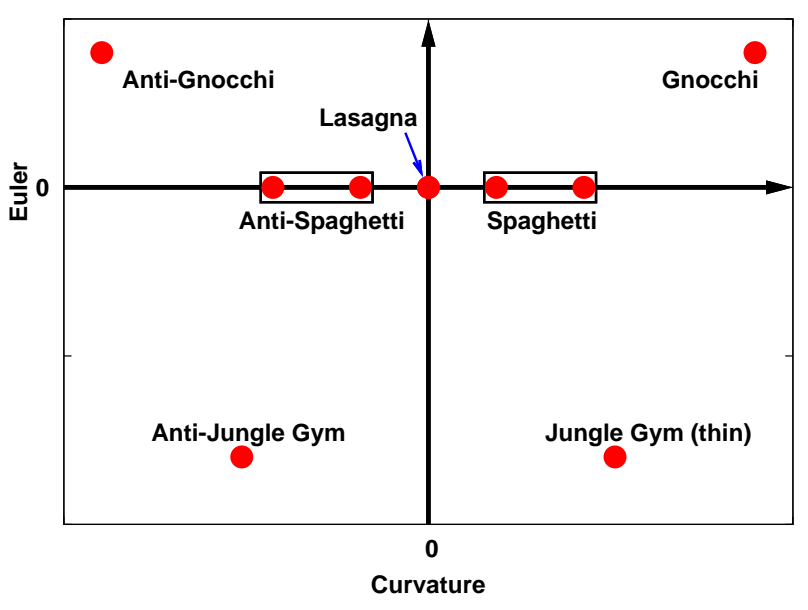

FIG. 13: (Color online) Systematic classification of the artificial structures in terms of the curvature and Euler number.

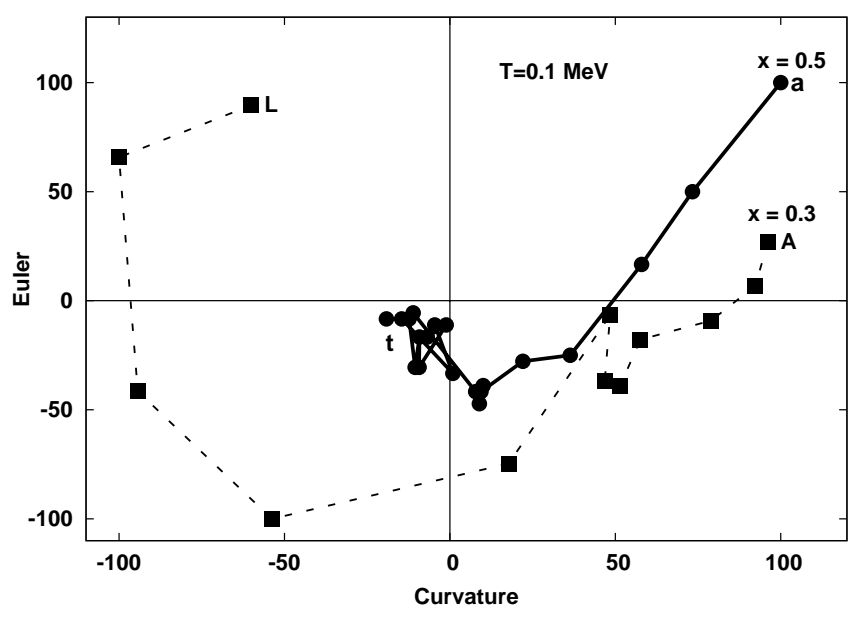

FIG. 14: Curvature-Euler coordinates of the structures listed in table \ Labels "A", "a", "L" and "t" correspond, respectively, to the initial and final points in the table.
In future investigations we will apply this method to an in-depth study of the origin of clustering. As hinted in [44], the role of the long range Coulomb interaction has not been fully explored; current exploratory runs are indicating that Coulomb merely shifts the space scales a bit but is not an "if-and-only-if" requirement for the formation of clusters. Along the same lines, as the present $C M D$ model can function both with a stiff and medium compressibility potentials, we also plan to investigate the role of the equations of state in the formation of the pasta structures.

\section{Acknowledgments}

C.O.D. is a member of the "Carrera del Investigador" CONICET supported by CONICET through grant PIP5969, and acknowledges the warm hospitality of the University of Texas at El Paso. J.A.L. Acknowledges support from grant nsf-phy 1066031, thanks Dr. Jorge Piekarewicz for suggesting the use of $C M D$ to study the nuclear pasta, Dr. L.G. Valdez Sánchez for clarifying observations about our use of topological objects, and the hospitality of the University of Buenos Aires and of the Lawrence Berkeley Laboratory where this manuscript was completed.
[1] P. Haensel and J.L. Zdunik, Astron. Astrophys. 229, 117 (1990)

[2] K. Oyamatsu, Nucl. Phys. A561, 431 (1993).

[3] G. Baym, H.A. Bethe and C. Pethick, Nucl. Phys. A175, 225 (1971).

[4] C.P. Lorenz, PhD Thesis, University of Illinois, unpublished (1991)

[5] F. Douchin and P. Haensel, Phys. Letters B485, 107 (2000).

[6] P. Ring and P. Schuck, The Nuclear Many-Body Problem, Springer-Verlag, New York (1980) (Chapter 13).

[7] M. Brack, C. Guet and H.-B. Håkansson, Phys. Rep. 123, 275 (1985).

[8] S.S. Avancini, C.C. Barros, Jr., D.P. Menezes and C. Providência, Phys. Rev. C82 025808 (2010).
[9] P. Magierski and P.H. Heenen, Phys.Rev. C65, 045804 (2002).

[10] D.G. Ravenhall, C.D. Bennett and C.J. Pethick, Phys. Rev. Lett. 28, 978 (1972).

[11] C.J. Pethick and D.G. Ravenhall: Ann. Rev. Nucl. Part. Sci. 45, 429 (1995).

[12] J.W. Negele and D. Vautherin, Nucl. Phys. A207, 298 (1973).

[13] W. Newton and J. Stone, Phys. Rev. C79 055801 (2009)

[14] C.J. Horowitz, M.A. Perez-Garcia, D.K. Berry and J. Piekarewicz, Phys. Rev. C72, 035801 (2005).

[15] C.J. Horowitz, M.A. Perez-Garcia, and J. Piekarewicz, Phys. Rev. C69, 045804 (2004).

[16] C.J. Horowitz, M.A. Perez-Garcia, J. Carriere, D.K. Berry, and J. Piekarewicz, Phys. Rev. C70, 065806 
(2004).

[17] T. Maruyama, K. Niita, K. Oyamatsu, T. Maruyama, S. Chiba and A. Iwamoto, Phys. Rev. C57, 655 (1998).

[18] K. Oyamatsu, M. Hashimoto and M. Yamada, Prog. Theor. Phys. 72373 (1984).

[19] G. Watanabe et al., Phys. Rev. Lett. 103121101 (2009).

[20] K. Nakazato, K. Oyamatsu and S. Yamada, Phys. Rev. Lett. 103132501 (2009).

[21] G. Watanabe, K. Sato, K. Yasuoka and T. Ebisuzaki, Phys. Rev. C66, 012801 (2002).

[22] G. Watanabe, K. Iida and K. Sato, Nucl. Phys. A676, 445 (2000).

[23] H. Sonoda, G. Watanabe, K. Sato, K. Yasuoka, and T. Ebisuzaki, Phys. Rev. C81, 049902(E) (2010).

[24] T. Maruyama, T. Tatsumi, D.N. Voskresensky, T. Tanigawa and S. Chiba, Phys. Rev. C72 015802 (2005) .

[25] O.L. Caballero, S. Postnikov, C.J. Horowitz and M. Prakash, Phys. Rev. C78, 045805 (2008).

[26] Débora P. Menezes, Sidney S. Avancini, Constança Providência, Marcelo D. Alloy, in "Neutron Star Crust", Eds. C.A. Bertulani and J. Piekarewicz, in print, Nova Science Publishers, Inc. (2012).

[27] C.J. Horowitz, Eur. Phys. J. A30, 303 (2006).

[28] K. Nakazato, K. Iida and K. Oyamatsu, Phys. Rev. C83 065811 (2011).

[29] H.W. Barz, J.P. Bondorf, D. Idier, and I.N. Mishustin, Phys. Lett. B382, 343 (1996).

[30] J.A. López and J. Randrup, Nuc. Phys. A571, 379 (1994); ibid, A503, 183-222, (1989); ibid, A491, 477491 (1989).

[31] E.A. Uehling and G.E. Uhlenbeck, Phys. Rev. 43, 552 (1933).

[32] A. Polanski et al., Radiat. Prot. Dosimetry, 115, (2005).

[33] J. Taruna, "The physics of compact stars", Ph.D. Thesis, Florida State University, AAT 3321532 (2008).

[34] A. Barrañón, C.O. Dorso, J.A. López and J. Morales, Rev. Mex. Fís. 45, 110 (1999).

[35] A. Vicentini, G. Jacucci and V. R. Pandharipande, Phys. Rev. C31, 1783 (1985); R. J. Lenk and V. R. Pandharipande, Phys. Rev. C34, 177 (1986); R.J. Lenk, T.J. Schlagel and V. R. Pandharipande, Phys. Rev. C42,372 (1990).
[36] A. Chernomoretz, L. Gingras, Y. Larochelle, L. Beaulieu, R. Roy, C. St-Pierre and C. O. Dorso, Phys. Rev. C65, 054613 (2002).

[37] A. Barrañón, C.O. Dorso and J.A. López, Rev. Mex. Fís. 47-Sup. 2, 93 (2001).

[38] A. Barrañón, C.O. Dorso, and J.A. López, Nuclear Phys. A791, 222 (2007).

[39] A. Barrañón, R. Cárdenas, C.O. Dorso, and J.A. López, Heavy Ion Phys. 17, 1, 41 (2003).

[40] C.O. Dorso and J.A. López, Phys. Rev. C64, 027602 (2001).

[41] A. Barrañón, J. Escamilla Roa and J.A. López, Braz. J. Phy., 34-3A 904 (2004).

[42] A. Barrañón, J. Escamilla Roa and J.A. López, Phys. Rev. C69, 014601 (2004).

[43] C.O. Dorso, C.R. Escudero, M. Ison and J.A. López, Phys. Rev. C73, 044601 (2006).

[44] C.O. Dorso, P.A. Giménez Molinelli and J.A. López, J. Phys. G: Nucl. Part. Phys. 38115101 (2011); ibid, Rev. Mex. Phys., S 57 (1), 14 (2011).

[45] G. Watanabe, K. Sato, K. Yasuoka and T. Ebisuzaki, Phys. Rev. C68, 035806 (2003).

[46] C.O. Dorso, P.A. Giménez Molinelli and J.A. López, in "Neutron Star Crust", Eds. C.A. Bertulani and J. Piekarewicz, in print, Nova Science Publishers, Inc. (2012)

[47] H.C. Andersen, J. Chem. Phys. 722384 (1980).

[48] C.O. Dorso and J. Aichelin, Phys. Lett. B345, 197 (1995).

[49] A. Strachan and C.O. Dorso, Phys. Rev. C55, 775 (1997); ibid, Phys. Rev. C56, 995 (1997).

[50] C.O. Dorso and J. Randrup, Phys. Lett. B301, 328 (1993).

[51] C.O. Dorso and A. Strachan, Rev. Mex. Fis. 41 Sup. 1, 96-108, (1995).

[52] J.A. López and C.O. Dorso, Lecture Notes on Phase Transformations in Nuclear Matter, World Scientific, Hackensack, NJ, USA, ISBN 978-981-02-4007-3 (2000).

[53] K. Michielsen and H. De Raedt, Phys. Reports 347, 461 (2001). 